\title{
Airway remodeling in asthma
}

Perspective

See related articles, pages 985-999.

\author{
Jack A. Elias, ${ }^{1}$ Zhou Zhu, ${ }^{1}$ Geoffrey Chupp, ${ }^{1}$ and Robert J. Homer ${ }^{2,3}$ \\ ${ }^{1}$ Section of Pulmonary and Critical Care Medicine, Department of Internal Medicine, \\ Yale University School of Medicine, New Haven, Connecticut 06520, USA \\ ${ }^{2}$ Pathology and Laboratory Medicine Service, Veterans Affairs-Connecticut Healthcare Systems, \\ West Haven, Connecticut 06516, USA \\ ${ }^{3}$ Department of Pathology, Yale University School of Medicine, New Haven, Connecticut 06520, USA
}

Address correspondence to: Jack A. Elias, Section of Pulmonary and Critical Care Medicine, Yale University School of Medicine, 105 LCI, 333 Cedar Street, New Haven, Connecticut 06520-8057, USA. Phone: (203) 785-2788; Fax: (203) 785-7146.

Asthma is estimated to affect 15 million people in the United States. After declining in the 1970s, its prevalence, morbidity, and mortality have increased since the 1980s. It now affects 1 in 7 children in the United Kingdom, and is the most frequent reason for preventable childhood hospitalizations in the United States. In 1990, total asthma-related health care costs were estimated at 6.2 billion dollars in the United States. This figure exceeded 10 billion dollars by 1995 .

Asthma used to be thought of as an entirely reversible disorder. As a consequence, our therapeutic approach to asthma has focused on symptomatic control through the alleviation of bronchospasm and the reduction of airway inflammation. It is now known, however, that this optimistic picture of the natural history of asthma is not entirely complete. A number of studies have demonstrated that asthmatics, as a group, experience an accelerated rate of respiratory functional deterioration $(1,2)$. This is nicely illustrated in a recent study of 17,506 people followed over a 15-year period in the Copenhagen Heart Study. In this population, the 1,095 patients with selfreported asthma experienced a greater decline in airway function (forced expiratory volume in 1 second [FEV1]) than did controls ( $38 \mathrm{~mL}$ per year vs. $22 \mathrm{~mL}$ per year), and the greatest rates of decline were experienced by smokers with asthma (1). Patient-to-patient variability has also been noted, with some patients acquiring a physiologic state characterized by irreversible or partially reversible airway obstruction, and others manifesting incomplete $\beta$-agonist responses and persistent airways hyperresponsiveness (AHR) even after prolonged corticosteroid therapy. The pathologic correlates and pathogenetic mechanisms responsible for these findings are incompletely understood. Recent attention has, however, been directed at the structural changes - collectively referred to as airway remodeling - in the asthmatic and normal airway. This is based on the belief that these changes are responsible, in a cause-and-effect fashion, for these adverse outcomes and for other manifestations of the asthmatic diathesis. The change that this represents in our concept of the pathogenesis of asthma and the features and pathogenesis of asthmatic airway remodeling is reviewed below.

\section{Evolving concepts of asthma pathogenesis}

Asthma used to be considered largely in terms of the abnormal contraction of airway smooth muscle (bronchospasm), which was presumed to be due to an intrinsic abnormality in the airway myocyte. Nervous system dys- function with cholinergic and/or tachykinin excess was also proposed as an important pathogenetic process. However, the prominence of inflammation in bronchoscopic biopsies obtained during periods of disease activity and clinical stability, the beneficial clinical effects of steroids, and postmortem studies demonstrating bronchial eosinophilic infiltrates (Figure 1) prompted a reevaluation of these concepts and an appreciation of the central role of inflammation in the asthmatic diathesis $(3,4)$. Studies using bronchoalveolar lavage (BAL) and bronchial biopsies have demonstrated that a variety of cells and mediators are involved, with IgE and mast cells implicated in the acute response, eosinophils and eosinophil granule proteins in the late response, and with $\mathrm{T}$ cells, particularly Th2 cells, orchestrating these responses through their production of cytokines such as IL-4, IL-5, IL-9, and IL-13 (refs. 5-8; also see other articles in this Perspective series). Recent studies have also implicated stromal and epithelial cells in the inflammatory response, as shown by the ability of these cells to respond to Th2 cytokines with the production of chemokines that initiate and perpetuate tissue inflammatory reactions. As a result, a pathogenetic construct for atopic asthma (and possibly other forms of asthma) has been put forth in which (a) Th2 cells play a central role in the recognition of antigens and the initiation and perpetuation of inflammation; (b) eosinophils are important proinflammatory and epithelial damaging cells; and (c) a variety of cells, including mast cells, epithelial cells, basophils, fibroblasts, smooth muscle cells, and macrophages, contribute through the secretion of cytokines that generate tissue inflammation and/or influence Th2 lymphocyte and/or eosinophil function.

Implicit in the "inflammation theory" of asthma is the belief that inflammation is both necessary and sufficient to account for the complex features of asthma. Although inflammation is undoubtedly a cornerstone of asthma, it is now clear that the asthmatic response is more complex. It is also clear from pathologic investigations that structural alterations exist in the asthmatic airway. Mathematical modeling studies have provided evidence that these alterations contribute to the symptoms and physiologic dysregulation seen in asthma (see below). As a result, it has been proposed that the chronic inflammation that is characteristic of the asthmatic airway leads to a remodeling response, and that the structural alterations caused by this response play an important role in generating the manifestations of the disorder. This conceptu- 
al evolution predicts that we will acquire an enhanced understanding of asthma pathogenesis when we think of asthma in the context of paradigms of injury and wound healing, as well as the traditional paradigms relevant to the interface of inflammation and airway physiology. It also suggests that studies utilizing this new perspective will identify novel targets against which therapies can be directed, and will provide an enhanced understanding of the biologic basis of the patient-to-patient variability that is encountered in this disorder.

\section{Remodeling responses in the asthmatic airway}

Airway remodeling is difficult to define. It can, however, be thought of as a collective term that encompasses the alterations in structural cells and tissues in the asthmatic, as opposed to the normal, airway. It was first appreciated over 75 years ago by Huber and Koessler in their classic description of fatal asthma (9). More recent pathologic and morphometric studies have clarified this response. Although these studies differ in the characteristics of their study subjects (including smoking status, atopy, and disease severity), definition of disease, methodologies, and choice of tissue (autopsy vs. biopsy), they have highlighted the wall thickening, subepithelial fibrosis, mucus metaplasia, myofibroblast hyperplasia, myocyte hyperplasia and hypertrophy, and epithelial hypertrophy characteristic of the remodeling response. Selected aspects of this response are reviewed below.

Airway wall thickening. All components of the airway wall (inner, outer, and total) have been reported to be thickened in asthma $(10,11)$. Many elements contribute to this response, including an increase in airway smooth muscle; edema; inflammatory cell infiltration; glandular hypertrophy; and connective tissue deposition. Airway wall thickness is increased $50-300 \%$ in cases of fatal asthma and $10-100 \%$ in cases of nonfatal asthma (10), compared with nonasthmatic controls. In fatal asthma, the thickening is most marked in the small cartilaginous and large membranous airways. In nonfatal asthma, the thickening is more marked in small membranous airways (diameter $<0.6 \mathrm{~mm}$ ) and mid-sized airways (diameter $1-3 \mathrm{~mm}$ ), and is less marked in the central airways. The physiologic and clinical consequences of airway wall thickening are incompletely understood. A number of studies suggest that increases in wall thickness correlate with disease severity. Mathematical modelings that incorporate morphologic and physiologic measure- ments made on asthmatic and nonasthmatic tissues (10, 12) have demonstrated that the thickening response can enhance the degree of luminal effacement caused by a given degree of muscle contraction, and that the magnitude of airway wall thickening is sufficient to contribute substantially to asthmatic AHR. They have also demonstrated that increased thickness of the outer wall could uncouple the airway from the distending and tethering forces of the surrounding lung parenchyma, thereby allowing greater degrees of smooth muscle contraction with less tension development and increased maximal muscle shortening. The recently popularized "perturbed equilibrium hypothesis" also suggests that wall thickening can destabilize the dynamic forces that control airway caliber, causing airway collapse (13). All in all, these studies suggest that wall thickening increases the airway narrowing caused by smooth muscle stimulation, thereby promoting bronchospasm and hyperresponsiveness to bronchospastic stimuli.

Subepithelial fibrosis. Early postmortem studies described what was believed to be thickening of the asthmatic basement membrane. Electron microscopy demonstrated that the true basement membrane, the lamina rara and densa, is normal in these tissues. The "thickening" is the result of a dense fibrotic response that occurs primarily in the lamina reticularis $(4,14,15)$. This response is characterized by the enhanced accumulation of fibronectin and types I, III, and V collagens. Type IV collagen and laminin are not present in enhanced quantities. The pathophysiologic significance of subepithelial fibrosis is not clear. However, the presence of subepithelial fibrosis has been associated with disease severity and correlated with a decline in $\operatorname{FEV}_{1}(16,17)$. In addition, significant correlations have been reported between methacholine sensitivity and the intensity of airway fibrosis $(18,19)$ and between subepithelial thickness and frequency and duration of asthma symptoms. These findings suggest that the fibrotic response may play a role in disease severity and in the generation of the incompletely reversible airway obstruction that is seen in some patients. They also suggest that fibrosis may lead to, or be a determinant or marker of, a pathway(s) that leads to AHR. Caution is required, however, because subepithelial fibrosis has been noted in biopsies from patients with mild asthma $(14,15)$ and in nonasthmatics with allergic rhinitis, and because patient populations have been reported in which asthma severity does not correlate with submucosal collagen deposition (20). Additional investigation will be required to determine if these conflicting observations address the consequences of subepithelial fibrosis or, instead, suggest that patients can acquire severe asthma through mechanisms that may or may not involve airway remodeling.

Increased myocyte muscle mass. An increase in the area of airway smooth muscle has been demonstrated in most, but not all, studies of fatal and nonfatal asthma (10). The changes that have been noted are most marked in the central airways in asthma fatali- 


\begin{tabular}{|c|c|c|c|c|c|c|}
\hline & \multicolumn{2}{|c|}{ IL-6 type } & \multicolumn{4}{|c|}{ Th2 type } \\
\hline & IL-6 & IL-11 & IL-13 & IL-4 & IL-9 & IL-5 \\
\hline \multicolumn{7}{|l|}{ 1.Inflammation } \\
\hline Lymphocytes & $+/-A$ & $+/-^{A}$ & ++ & ++ & ++ & ++ \\
\hline Eosinophils & - & - & ++ & ++ & ++ & +++ \\
\hline 2. Subepithelial fibrosis & +++ & +++ & ++ & $+/-$ & $+/-$ & ++ \\
\hline 3. Myocyte hyperplasia & $?$ & ++ & - & - & $?-$ & $?$ \\
\hline 5. Mucus metaplasia & - & - & +++ & +++ & +++ & ++ \\
\hline 6. Effect on antigen-induced inflammation & Inhibit & Inhibit & $?$ & $?$ & $?$ & $?$ \\
\hline 7. Airway obstruction & - & Present & Present & Present & - & $?$ \\
\hline 8. AHR & - & Present & Present & - & Present & Present \\
\hline
\end{tabular}

Tabular summary from our studies and the literature of remodeling-relevant responses in transgenic overexpression mice in which the CC10 promoter was used to overexpress cytokines in the airway. Comparisons are made (when possible) of the magnitude of airway inflammatory infiltration with lymphocytes and eosinophils, airway fibrosis, myocyte hyperplasia, myofibroblast hyperplasia, and mucus metaplasia. The effects of the overexpression of these cytokines on antigen- or ovalbumin-driven tissue inflammation are also highlighted. +, positive/present weak effect; ++, positive/present moderate effect; +++, positive/present strong effect; -, not present; ?, unknown;

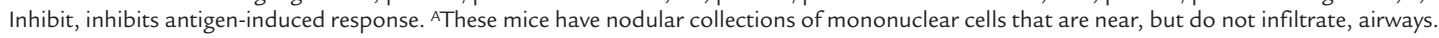

ties and in the large membranous bronchioles in the nonfatal specimens. Some investigators believe that hyperplasia is the major contributor to this response (21). Others have described 2 distinct responses (22). In type I asthma, the increase in muscle mass was associated only with hyperplasia and was restricted to large central airways. In type II asthma, comparatively mild hyperplasia was seen in the large airways, and hypertrophy was detected throughout the bronchial tree, particularly in small peripheral airways (22). It is not clear if these different patterns reflect different types of injuries or different tissue or host response mechanisms. It is clear from modeling studies that either abnormality can contribute to the AHR and bronchospasm characteristic of the asthmatic diathesis $(10,12)$.

Myofibroblast hyperplasia. Subepithelial myofibroblast hyperplasia has been repeatedly demonstrated in asthma and models of asthmatic responses (23-25). Early studies identified these cells using antibodies against colonic pericrypt sheath cells. Subsequently, $\alpha$-smooth muscle actin-negative and -positive myofibroblasts have been documented. The origin and fate of these cells is not fully understood. Cells with this phenotype appear quickly after antigen challenge, possibly implying a quiescent precursor cell that acquires myofibroblastic features without dividing (25). Myofibroblasts are major producers of collagenous and noncollagenous matrix molecules. They may serve a similar role in asthma, because the number of myofibroblasts present in the submucosa correlates with subepithelial collagen deposition (24).

Mucus metaplasia. Mucus hypersecretion, epithelial hypertrophy, mucus metaplasia, and airway obstruction due to bronchial mucus plugging are well-documented features of chronic asthma and status asthmaticus (26, 27). In humans, subepithelial glands are the major source of airway mucus. Morphometric studies demonstrated that the area of mucus glands is increased in fatal and nonfatal asthma. Similarly, the area of the airway epithelium is increased in asthmatic airways (10), and mucus occupies a higher percentage of the lumen of the central airways in asthma fatalities than in controls (26). Epithelial hypertrophy and airway mucus can contribute to the airway obstruction seen in asthma. Liquid and mucus on the airway surface can also amplify the effects of muscle shortening and increase the surface tension at the air-liquid interface, thereby adding to the forces that tend to narrow the airway.

\section{Mediators and mechanisms of asthmatic remodeling}

The stimuli that induce airway remodeling are not well defined. A number of factors contribute to this lack of knowledge, including the limited availability of tissues from patients with remodeled airways, the difficulties that are inherent in attempts to define mechanisms of chronic responses from data obtained at a single point(s) in time, and the problematic nature of attempts to differentiate cytokines and mediators that are involved in chronic and acute airway responses. This is compounded by the complex relationship between inflammation and remodeling, with chronic inflammation inducing remodeling responses in some settings but not in others. Although subepithelial fibrosis is only one of the features of the remodeled airway, it has received the most attention. These studies suggest that TGF- $\beta$ family proteins play an important role in this response, because (a) they are detected in exaggerated quantities in asthmatic BAL fluid before and after antigen challenge; (b) they are produced in exaggerated quantities by eosinophils and fibroblasts from patients with severe and mild asthma, as well as by those from controls; and (c) in most, but not all, studies, TGF- $\beta_{1}$ expression correlates with basement membrane thickness and fibroblast number and/or disease severity $(16,28,29)$. GM-CSF and EGF are also found in exaggerated quantities in airway biopsies from patients with asthma (28), and the overexpression of GM-CSF causes pulmonary inflammation with eosinophilia and fibrosis. In limited investigations, however, neither GM-CSF nor EGF has been demonstrated to correlate with parameters of airway fibrosis (28), raising the possibility that these mediators are involved in acute, but not chronic, airway responses. Studies in other systems have demonstrated that a variety of mediators have the ability to regu- 
late fibroblast proliferation and matrix production, including PDGF, IL-1, TNF, IL-5, IGF-1, endothelin-1, tryptases, and leukotrienes. The effects of these agents can be cell- and microenvironment-specific. Similarly, large and partially overlapping lists of mediators have been implicated in the regulation of other facets of airway remodeling. The relevance of these mediators to human asthma will require additional investigation.

Fibrosis is a dynamic process that involves the balance of matrix metalloproteinases (MMPs) and their inhibitors, the tissue inhibitors of metalloproteinases (TIMPs). MMP-9 is found in exaggerated quantities in sputum and biopsies from patients with asthma $(30,31)$. MMP-8 is the major MMP found at sites of normal wound healing, and is produced and activated in an exaggerated fashion in nonhealing wounds - an intriguing paradigm for the asthmatic airway. TIMP-1 is also present in exaggerated quantities in sputum and biopsies from patients with asthma. The levels of TIMP-1 correlate with asthmatic airway obstruction, and the exaggerated production of TIMP-1 may participate in the generation of asthmatic airway fibrosis $(31,32)$. In addition, in some reversible fibrotic disorders, the resolution of fibrosis is temporally associated with a marked decrease in TIMP-1 and TIMP-2 expression.

\section{Lessons from transgenic mice}

The use of wild-type animals to model airway remodeling is in its infancy. Only recently have protocols been described that adequately reproduce features of virusinduced (33) and antigen-induced (34) remodeling responses in rodents. In contrast, genetically modified mice have already provided important insights. Particularly significant information has been obtained from mice in which mediators relevant to chronic asthmatic inflammation are overexpressed in the lung using the Clara cell $10-\mathrm{kDa}$ (CC10) protein promoter. The types of information that have been obtained can be appreciated from studies of the IL-6-type and Th2 cytokines reviewed below (Table 1 ).

IL-6-type cytokines. The IL-6-type cytokine family contains IL-6, IL-11, cardiotropin-1, oncostatin-M, and ciliary neurotropic factor. These cytokines have overlapping effector profiles, similar 3-dimensional structures, and all use the promiscuous gp130 molecule in their multimeric receptor complexes.

IL-6. IL-6 is a pleiotropic molecule that has been repeatedly found in exaggerated quantities in biologic fluids and tissues from patients with asthma and models of the asthmatic diathesis. Its ability to stimulate $T$ and B cells and contribute to the development of Th2dominated inflammation led to the speculation that it plays a role in the generation and/or perpetuation of asthmatic tissue inflammation. However, IL-6 also has anti-inflammatory and cytoprotective activities, and is the major stimulator of the acute-phase response. This led to the belief that IL- 6 may also be a general-alarm signal that marshals protective responses that serve to diminish tissue inflammation and injury. An indication that IL-6 might participate in airway remodeling came from studies from our laboratories that used the CC10 promoter to overexpress IL-6 in the murine airway (35).
These and subsequent studies demonstrated that IL-6 causes impressive subepithelial fibrosis, with collagen deposition and the enhanced accumulation of $\alpha$-smooth muscle actin-containing stromal cells (Table 1). IL-6 did not cause tissue eosinophilia, mucus metaplasia, or AHR upon methacholine challenge.

IL-11. IL-11 is a multifunctional molecule that stimulates thrombopoiesis, induces the production of TIMP1 , and regulates bone metabolism. Studies from our laboratory demonstrated that IL-11 is produced by a variety of lung stromal cells, including airway and alveolar epithelial cells, fibroblasts, and airway myocytes, in response to TGF- $\beta$, IL-1, asthma-associated viruses, histamine, and eosinophil major basic protein $(36,37)$. Our studies also demonstrated that the transgenic overexpression of IL-11 causes an airway phenotype characterized by (a) subepithelial fibrosis; (b) the enhanced deposition of types III and I, but not type IV, collagens; (c) the enhanced accumulation of fibroblasts, myofibroblasts, and myocytes; and (d) baseline airway obstruction and AHR upon methacholine challenge (23). Tissue eosinophilia and mucus metaplasia are not features of the lungs of these animals. The validity of the transgenic approach was confirmed in studies that applied immunohistochemistry and in situ hybridization to biopsies from human asthmatics (38). These investigations demonstrated that IL-11 mRNA and protein can be detected in large quantities in biopsies from patients with severe asthma, but not in biopsies from patients with mild asthma or from normals. In these tissues, IL11 is expressed in epithelial cells and eosinophils. Further insight was obtained from studies utilizing the IL11-overexpressing transgenic mice in models of airway inflammation and lung injury. These studies demonstrated that IL-11 inhibits the acute eosinophilic response and Th2 gene expression induced by sensitization and challenge with the aeroallergen ovalbumin. When viewed together, the fibrogenic and anti-inflammatory effects of IL-11 suggest that IL-11 can be a healing molecule in the airway.

Th2 cytokines. Th2-dominated inflammation is a cornerstone of the asthmatic diathesis. It is thus tempting to speculate that Th2 cytokines play a key role in asthmatic airway remodeling. Transgenic overexpression studies from our laboratories and others' provide in vivo support for this contention. The CC10-driven overexpression of IL-13 in the lung/airway caused a profound phenotype characterized by eosinophilic and mononuclear inflammation, with mucus metaplasia, subepithelial fibrosis, airway obstruction, and AHR (39) (Table 1). Similarly, the overexpression of IL-9 and IL-4 caused a mixed mononuclear and eosinophilic inflammatory response $(40,41)$, and IL-5 caused impressive tissue eosinophilia (42). IL-4, IL-5, and IL-9 caused extensive mucus metaplasia, and IL-9 and IL-5 caused subepithelial fibrosis and AHR (40-42). The data on IL-4 is less impressive, with a mild fibrotic response being reported by some, but not all, investigators $(41,43)$. At present, prominent muscle changes have not been described in any of these Th2 cytokine-overexpression systems.

Summary: transgenic overexpression modeling. Transgenic 
overexpression modeling has proven to be a powerful method for defining and comparing the in vivo effector functions of asthma-relevant molecules. An adequate number of mediators have now been studied using this methodology to allow remodeling paradigms to begin to be elucidated. For example, the IL-6-type cytokines cause prominent airway fibrosis, with prominent myocyte and myofibroblast abnormalities. In the case of IL-11, airway obstruction and AHR are also noted. Airway eosinophilia and mucus metaplasia are not induced by these moieties. In contrast, the Th2-type cytokines cause mixed eosinophilic and mononuclear inflammatory responses, prominent mucus metaplasia, and less impressive airway fibrosis. In addition, smooth muscle abnormalities have not been described. These observations identify candidate molecules that might mediate the individual manifestations of the remodeling response. More importantly, they demonstrate that, to date, no single mediator has been able to recapitulate all of the features described in remodeled asthmatic airways. This suggests that the remodeling response in the asthmatic airway is generated through the concerted actions of multiple mediators, and that a variety of interventions will be required to modulate the many facets of this complex tissue reaction.

\section{The course of remodeling responses in the airway}

Remodeling responses in asthma are now being considered as potential targets for therapeutic intervention. The utility of this approach, however, will depend on a number of factors. One centers on our ability to differentiate patients likely to develop clinically significant remodeling responses from those unlikely to develop such responses. The validation of biomarkers that predict airway remodeling would allow us to select and treat high-risk populations while avoiding side effects induced by unnecessary treatment in low-risk populations. To date, the utility of inflammatory biomarkers in predicting the development of chronic structural changes in the airway has not been substantiated. In addition, surprisingly little is known about the utility of remodeling-relevant biomarkers - such as the levels (in sputum, BAL, or tissues) of collagen-degradation peptides, hyaluronic acid, and isoforms of laminin, fibronectin, and tenascin - even though hyaluronic acid levels correlate with the chronicity of asthmatic inflammation (44), and laminin, fibronectin, and tenascin are markers of repair in asthmatic tissues.

Knowledge of the reversibility of the airway remodeling phenotype is also essential. Surprisingly, the natural history of airway remodeling is poorly understood. Some studies have demonstrated that subepithelial fibrosis is unchanged over time, both in the presence and absence of steroid therapy. This has led to the statement that the structural changes, including muscle alterations and fibrosis, are "essentially irreversible" (45). However, others have reported that steroids decrease subepithelial type III collagen deposition. In addition, after cessation of exposure, patients with isocyanate-induced asthma manifest decreases in subepithelial fibrosis and in fibroblast, mast cell, and lymphocyte numbers (46). It is not known if these divergent results reflect different types of injuries, different mechanisms of tissue remodeling, or variations in host response.

\section{Future directions}

In recent years, we have gone from the belief that there is an intrinsic abnormality in the airway myocyte to the belief that inflammation is the cornerstone of the asthmatic diathesis. We are now entertaining the hypothesis that aspects of the asthmatic phenotype are the result of tissue remodeling responses and structural alterations in the airway. This concept represents an important and exciting refinement in our thinking. It will, however, need further testing and evaluation. We need to know what contribution each of the features of the remodeled airway makes to the symptoms, abnormal physiology, and natural history of asthma. Particular attention needs to be directed to the definition and identification of subpopulations of asthmatics in which these manifestations may be particularly important. We also need to know what types of interventions alter the various features of the remodeling response and what effects these interventions have on the manifestations of the disease. These investigations will be aided greatly by the validation of biomarkers that predict which patients are at greatest risk for developing the different features of the airway remodeling response. The cellular and molecular events involved in the pathogenesis of the various features of the asthmatic airway also need to be defined. An important facet of these studies will be attempts to determine the extent to which these responses represent healing and repair responses and/or aspects of disease pathogenesis, because, in theory, healing and repair responses may be able to be initiated earlier and/or augmented to confer benefit to the patient. Lastly, studies need to be directed at the cellular, molecular, and genetic factors that are responsible for determining why only some people develop significant remodeling and why the type of remodeling that occurs can differ from patient to patient. The information that will come from these and other studies will profoundly effect our understanding of asthma pathogenesis, and will impact the types of strategies we employ to combat this increasingly problematic disorder.

\section{Acknowledgments}

This work was supported by National Institutes of Health grants R01 HL-36708, P50 HL-56389, RO1 HL61904, and K08 HL-04007.

\footnotetext{
1. Lange, P., Parner, J., Vestbo, J., Schnohr, P., and Jensen, G. 1998. A 15-year follow-up study of ventilatory function in adults with asthma. N. Engl.J. Med. 339:1194-1200.

2. Peat, J.K., Woolcock, A.J., and Cullen, K. 1987. Rate of decline of lung function in subjects with asthma. Eur. J. Respir. Dis. 70:171-179.

3. Holgate, S.T., Wilson, J.R., and Howarth, P.H. 1992. New insights into airway inflammation by endobronchial biopsy. Am. Rev. Respir. Dis. 145:S2-S6.

4. Bradding, P., Redington, A.E., and Holgate, S.T. 1997. Airway wall remodelling in the pathogenesis of asthma: cytokine expression in the airways. In Airway wall remodelling in asthma. A.G. Stewart, editor. CRC Press. Boca Raton, FL. 29-63.

5. Robinson, D.S., et al. 1992. Predominant Th2-like bronchoalveolar Tlymphocyte population in atopic asthma. N. Engl. J. Med. 326:298-304.

6. Wills-Karp, M., et al. 1998. Interleukin-13: central mediator of allergic asthma. Science. 282:2258-2260.

7. Nicolaides, N.C., et al. 1997. Interleukin 9: a candidate gene for asthma. Proc. Natl. Acad. Sci. USA. 94:13175-13180.

8. Hamid, Q., et al. 1991. Expression of mRNA for interleukin-5 in mucos-
} 
al bronchial biopsies from asthma. J. Clin. Invest. 87:1541-1546.

9. Huber, H.L., and Koessler, K.K. 1922. The pathology of bronchial asthma. Arch. Intern. Med. 30:689-760.

10. James, A.J. 1997. Relationship between airway wall thickness and airway hyperresponsiveness. In Airway wall remodelling in asthma. A.G. Stewart, editor. CRC Press. Boca Raton, FL. 1-27.

11. Kuwano, K., et al. 1993. Small airways dimensions in asthma and chronic obstructive pulmonary disease. Am. Rev. Respir. Dis. 148:1220-1225.

12. Pare, P.D., Roberts, C.R., Bai, T.R., and Wiggs, B.J. 1997. The functional consequences of airway remodelling in asthma. Monaldi Arch. Chest Dis. 52:589-598.

13. Fredberg, J.J. 1998. Airway smooth muscle in asthma: flirting with disaster. Eur. Respir. J. 12:1252-1256.

14. Jeffery, P.K., et al. 1992. Effects of treatment on airway inflammation and thickness of basement membrane reticular collagen in asthma: a quantitative light and electron microscopic study. Am. Rev. Respir. Dis. 145:890-899.

15. Roche, W.R., Williams, J.H., Beasley, R., and Holgate, S.T. 1989. Subepithelial fibrosis in the bronchi of asthmatics. Lancet. 1:520-524.

16. Minshall, E.M., et al. 1997. Eosinophil-associated TGF-beta1 mRNA expression and airways fibrosis in bronchial asthma. Am.J. Respir. Cell Mol. Biol. 17:326-333.

17. Chetta, A., et al. 1997. Airways remodeling is a distinctive feature of asth$\mathrm{ma}$ and is related to severity of disease. Chest. 111:852-857.

18. Boulet, L.-P., Belanger, M., and Carrier, G. 1995. Airway responsiveness and bronchial-wall thickening in asthma with or without fixed airflow obstruction. Am. J. Respir. Crit. Care Med. 152:865-871.

19. Hoshino, M., Nakamura, Y., and Sim, J.J. 1998. Expression of growth factors and remodelling of the airway wall in bronchial asthma. Thorax. 53:21-27.

20. Chu, H.W., et al. 1998. Collagen deposition in large airways may not differentiate severe asthma from milder forms of the disease. Am. J. Respir. Crit. Care Med. 158:1936-1944.

21. Heard, B.E., and Hossain, S. 1973. Hyperplasia of bronchial muscle in asthma. J. Pathol. 110:319-331.

22. Ebina, M., Takahashi, T., Chiba, T., and Motomiya, M. 1993. Cellular hypertrophy and hyperplasia of airway smooth muscles underlying bronchial asthma. A 3-D morphometric study. Am. Rev. Respir. Dis. 48:720-726.

23. Tang, W., et al. 1996. Targeted expression of IL-11 in the murine airway causes airways obstruction, bronchial remodeling and lymphocytic inflammation. J. Clin. Invest. 98:2845-2853.

24. Brewster, C.E.P., et al. 1990. Myofibroblasts and subepithelial fibrosis in bronchial asthma. Am. J. Respir. Cell Mol. Biol. 3:507-511.

25. Gizycki, M.J., Adelroth, E., Rogers, A.V., O’Byrne, P.M., and Jeffery, P.K. 1997. Myofibroblast involvement in the allergen-induced late response in mild atopic asthma. Am. J. Respir. Cell Mol. Biol. 16:664-673.

26. Aikawa, T., Shimura, S., Sasaki, H., Ebina, M., and Takashima, T. 1992. Marked goblet cell hyperplasia with mucus accumulation in the airways of patients who died of severe acute asthma attack. Chest. 101:916-921.

27. Kim, K.C., et al. 1997. Airway goblet cell mucin: its structure and regulation of secretion. Eur. Respir. J. 10:2644-2649.

28. Vignola, A.M., et al. 1997. Transforming growth factor- $\beta$ expression in mucosal biopsies in asthma and chronic bronchitis. Am. J. Respir. Crit. Care Med. 156:591-599.
29. Ohno, I., et al. 1996. Transforming growth factor beta 1 (TGF beta 1) gene expression by eosinophils in asthmatic airway inflammation. Am. J. Respir. Cell Mol. Biol. 15:404-409.

30. Vignola, A.M., et al. 1998. Sputum metalloproteinase-9/tissue inhibitor of metalloproteinase- 1 ratio correlates with airflow obstruction in asthma and chronic bronchitis. Am. J. Respir. Crit. Care Med. 158:1945-1950.

31. Hoshino, M., Nakamura, Y., Sim, J., Shimojo, J., and Isogai, S. 1998. Bronchial subepithelial fibrosis and expression of matrix metalloproteinase-9 in asthmatic airway inflammation. J. Allergy Clin. Immunol. 102:783-788

32. Mautino, G., et al. 1999. Increased expression of tissue inhibitor of metalloproteinase-1 and loss of correlation with matrix metalloproteinase9 by macrophages in asthma. Lab. Invest. 79:39-47.

33. Uhl, E.W., et al. 1996. Parainfluenza virus-induced persistence of airway inflammation, fibrosis, and dysfunction associated with TGF-beta 1 expression in brown Norway rats. Am. J. Respir. Crit. Care Med. 154:1834-1842.

34. Blyth, D.I., Pedrick, M.S., Savage, T.J., Hessel, E.M., and Fattah, D. 1996. Lung inflammation and epithelial changes in a murine model of atopic asthma. Am. J. Respir. Cell Mol. Biol. 14:425-438.

35. DiCosmo, B.F., et al. 1994. Airway targeted interleukin-6 in transgenic mice: uncoupling of airway inflammation and bronchial hyperreactivity. J. Clin. Invest. 94:2028-2035.

36. Leng, S., and Elias, J.A. 1997. Interleukin-11. Int. J. Biochem. Cell Biol. 29:1059-1062.

37. Einarsson, O., Geba, G.P., Zhu, Z., Landry, M., and Elias, J.A. 1996. Interleukin-11: stimulation in vivo and in vitro by respiratory viruses and induction of airways hyperresponsiveness. J. Clin. Invest. 97:915-924.

38. Minshall, E.M., et al. 1999. Interleukin $11 \mathrm{mRNA}$ expression is increased in severe asthma: localization to eosinophils. J. Allergy Clin. Immunol. 103:S58. (Abstr.)

39. Zhu, Z., et al. 1999. Pulmonary expression of interleukin-13 causes inflammation, mucus hypersecretion, subepithelial fibrosis, physiologic abnormalities and eotaxin production. J. Clin. Invest. 103:779-788.

40. Temann, U.-A., Geba, G.P., Rankin, J.A., and Flavell, R.A. 1998. Expression of interleukin 9 in the lungs of transgenic mice causes airway inflammation, mast cell hyperplasia and bronchial hyperresponsiveness. J. Exp. Med. 188:1307-1320.

41. Rankin, J.A., et al. 1996. Phenotypic and physiologic characterization of transgenic mice expressing interleukin 4 in the lung: lymphocytic and eosinophilic inflammation without airway hyperreactivity. Proc. Natl. Acad. Sci. USA. 93:7821-7825.

42. Lee, J.J., et al. 1997. Interleukin-5 expression in the lung epithelium of transgenic mice leads to pulmonary changes pathognomonic of asthma. J. Exp. Med. 185:2143-2156.

43. Jain-Vora, S., Wert, S.E., Temann, U.-A., Rankin, J.A., and Whitsett, J.A. 1997. Interleukin-4 alters epithelial cell differentiation and surfactant homeostasis in the postnatal mouse lung. Am. J. Respir. Cell Mol. Biol. 17:541-551.

44. Vignola, A.M., et al. 1998. Airway inflammation in mild intermittent and in persistent asthma. Am. J. Respir. Crit. Care Med. 157:403-409.

45. Barnes, P.J. 1996. Pathophysiology of asthma. Br. J. Clin. Pharmacol. 42:3-10.

46. Saetta, M. 1995. Airway wall remodeling after cessation of exposure to isocyanates in sensitized asthmatic subjects. Am. J. Respir. Crit. Care Med. 151:489-494. 